\title{
Análisis térmico de un disipador de calor con tubos de calor para procesadores de alto rendimiento
}

\section{(Thermal Analysis of heat sink with Heat Pipes for High Performance Processors)}

\author{
Fernando Toapanta Ramos ${ }^{1}$, Cristian Andrade², Emilio Davalos Álvarez³, Sebastián Landázuri \\ Zaldumbide ${ }^{4}$, William Quitiaquez ${ }^{5}$
}

\section{Resumen}

El objetivo de este documento es realizar el análisis térmico de un dispositivo disipador de calor por medio del uso de tubos de calor que contienen diferentes fluidos que son: agua, refrigerante R134a y metanol para la evaluación de estos dispositivos bajo ciertas características de diseño debido a que, en la actualidad, los procesadores tienen un mayor consumo energético y mayores capacidades de procesamiento, lo cual provoca una elevación significativa de la temperatura ante cargas de trabajo demandantes. Mediante el uso del software de simulación ANSYS, se realizó el estudio térmico del dispositivo; además, el gradiente de temperatura que se genera en el mismo al estar en contacto con una superficie caliente, la cual va a ser un procesador de alto desempeño, demostrando así que se puede obtener temperaturas estables a partir del uso de tubos de calor ante cargas de trabajo demandantes, asegurando un correcto funcionamiento y enfriamiento del procesador.

\section{Palabras clave}

Disipador; análisis térmico; tubo de calor; transferencia de calor; ANSYS.

\begin{abstract}
The objective of this document is to propose the thermal analysis of a heat dissipating device by using heat pipes containing different fluids, which are water, refrigerant R134a and methanol for the evaluation of these devices under certain design characteristics, due to the fact that at present the processors have a higher energy consumption and greater processing capacities, which causes a significant elevation of the temperature before demanding workloads. Through the use of the ANSYS simulation software, the thermal study of the device was carried out; in addition, the temperature gradient generated in it when in contact with a hot surface, which is going to be a high performance processor, demonstrating that stable temperatures can be obtained through the use of heat pipes at demanding workloads, ensuring correct operation and cooling of the processor.
\end{abstract}

\section{Keywords}

Heatsink; thermal analysis; heat pipe; heat transfer; ANSYS

\section{Introducción}

Existen dos métodos para lograr esta reducción en forma de intercambiadores de calor o tubos de calor (Chan, Siqueiros, Ling-Chin, Royapoor, y Roskilly, 2015). Los tubos de calor pueden ser una herramienta altamente efectiva para administrar y controlar térmicamente procesos que sean exotérmicos o endotérmicos, ya que proporcionan: a) separación de la fuente de calor y el disipador de calor, b) mantener la temperatura uniforme y constante; y, c) control de tempera-

$1 \quad$ Universidad Politécnica Salesiana, Grupo de Investigación en Energías Renovables e Implementación Mecánica de pymes (GIERIMP), Quito-Ecuador (Itoapanta@ups.edu.ec), https://orcid.org/0000-0002-0838-4702.

2 Universidad Politécnica Salesiana, Quito-Ecuador (candradet@est.ups.edu.ec)

3 Universidad Politécnica Salesiana, Quito-Ecuador (edavalosa@est.ups.edu.ec).

$4 \quad$ Universidad Politécnica Salesiana, Quito-Ecuador (dlandazurizaest.ups.edu.ec).

$5 \quad$ Universidad Politécnica Salesiana, Quito-Ecuador (wquitiaquez@ups.edu.ec). 
tura, es decir, tiempo de respuesta rápido (Blet, Lips, y Sartre, 2017; Jafari, Franco, Filippeschi, y Di Marco, 2016; Jafari, Shamsi, Filippeschi, Di Marco, y Franco, 2017; Khalifa, Tan, Date, y Akbarzadeh, 2015; Reay y Harvey, 2013; Shabgard et al., 2015).

Un tubo de calor es un dispositivo térmico utilizado para transferir el calor de forma pasiva de una posición a otra. En su forma básica es un tubo metálico que está sellado en ambos extremos y está parcialmente lleno con un fluido a presión de vacío. Los tubos de calor tienen una conductividad térmica efectiva muy alta, por lo tanto, se utilizan para transferir calor a distancias relativamente largas con una resistencia térmica mínima (Orr, Akbarzadeh, Mochizuki, y Singh, 2016). La tubería de calor se puede dividir en tres secciones: un evaporador, una sección adiabática y una sección de condensador. En la sección del evaporador, el tubo de calor recibe el calor y el fluido de trabajo se evapora. El vapor generado fluye a la sección del condensador, donde el calor se transfiere. Dado que el calor latente de evaporación y condensación del fluido de trabajo se utiliza dentro del tubo de calor, se puede transportar una cantidad relativamente grande de calor con una pequeña caída de temperatura de extremo a extremo. Además, la estructura de mecha bombea el condensado de vuelta a la sección del evaporador y, por lo tanto, la tubería de calor funciona continuamente sin consumir energía externa. Los tubos de calor se han utilizado ampliamente, especialmente para la refrigeración y la gestión térmica (Dillig, Leimert, y Karl, 2014; Faghri, 2014).

El desarrollo actual de las tecnologías en equipos electrónicos dentro del hardware computacional ha logrado producir procesadores cada vez más poderosos, que determinan el régimen de la vida cotidiana. Con los procesadores más avanzados, esto se ve reflejada en el incremento en la generación de calor, este calor se debe, principalmente, a su alta frecuencia y a la alta densidad de los transistores en el integrado (Garro et al., 2012). De la misma forma Lee, Pandiyan, Seo, Phelan, y Wu (2016) mencionan que, la distribución del calor de las plataformas informáticas ofrece una oportunidad interesante para la optimización del calor y la energía, tanto en las dimensiones horizontales como verticales. Con la miniaturización de los chips, la mejora de la capacidad de enfriamiento de la electrónica se vuelve esencial. De hecho, las densidades de flujo de calor aumentan cada vez más y no pueden ser disipadas por sistemas estándar basados en la convección de aire (Blet et al., 2017).

La conductividad térmica es una variable indispensable en los dispositivos de transferencia de calor, por esta razón el poseer dispositivos que midan y las estrategias que controlen esta variable en los procesos de enfriamiento y calentamiento son indispensables (Garc, 2018; Velasco Roldán, Goyos Pérez, Delgado García, y Freire Amores, 2017). El enfriamiento eficiente de dispositivos electrónicos y optoelectrónicos es de vital importancia para su operación exitosa y su larga vida útil. Los aumentos futuros en la velocidad, la potencia y la capacidad de tales dispositivos darán como resultado necesidades de disipación de calor cada vez más exigentes (Chen, Ye, Fan, Ren, y Zhang, 2016; Hung y Seng, 2011)

Para una óptima selección del sistema de almacenamiento de calor, los fluidos de cambio de fase y la geometría de la unidad de almacenamiento de calor desempeñan un papel crucial, mientras que la geometría del sistema de almacenamiento de calor impulsa la transferencia de calor. Los diferentes diseños geométricos de la unidad de almacenamiento térmico son cilíndricos o rectangulares (Dinker, Agarwal, y Agarwal, 2017).

En los trabajos realizados por Tang et al. (2018) enfatizan que los estudios actuales revelan que la capacidad de transferencia de calor máxima de los microtubos de calor ultrafinos disminuye con una reducción del grosor aplanado. Las variaciones de volumen en el canal de 
flujo de vapor y líquido causadas por el grosor aplanado son las razones principales del bajo rendimiento térmico. Los tubos de calor convencionales se han utilizado ampliamente para el enfriamiento de dispositivos electrónicos. Sin embargo, para los dispositivos electrónicos delgados y flexibles, actualmente en desarrollo, se necesita un nuevo dispositivo de enfriamiento debido a que los tubos de calor convencionales ofrecen una flexibilidad limitada en términos de forma debido a la estructura de la mecha (Lee y Kim, 2017).

Los fluidos de trabajo de los tubos de calor son los componentes esenciales que definen el rendimiento térmico. En general, el agua debe ser la mejor opción para enfriar los componentes electrónicos porque tiene el mayor calor latente y un punto de ebullición adecuado; sin embargo, otros fluidos también deben ser considerados seriamente (Chen et al., 2016). Para la selección del fluido de trabajo, la tensión superficial es una propiedad importante. Una mayor tensión superficial aumentará el efecto capilar y debe ser químicamente estable en presencia de la mecha (Chan et al., 2015).

Este trabajo se enfoca en realizar un estudio numérico y térmico, mediante dinámica de fluidos computacional (CFD) utilizando el software comercial ANSYS, de un dispositivo disipador de calor de cobre y aluminio con tubos de calor; además de utilizar varios fluidos como: agua, R134a y metanol; con la finalidad de realizar un análisis para la optimización de temperaturas de procesadores computacionales modernos, mediante la transferencia de calor por convección y conducción en el dispositivo.

\section{Metodología}

\subsection{Descripción de análisis}

En este trabajo se realizan simulaciones numéricas con la ayuda del software computacional ANSYS para determinar el comportamiento del dispositivo. En el estudio se toma como fluidos de trabajo dentro de los tubos de calor los siguientes: agua, refrigerante 134a y metanol; debido a su compatibilidad con el dispositivo y buen rendimiento térmico. En la Figura 1 se visualiza los componentes del disipador de calor que se está analizando.

Figura 1. Esquema general del disipador de calor

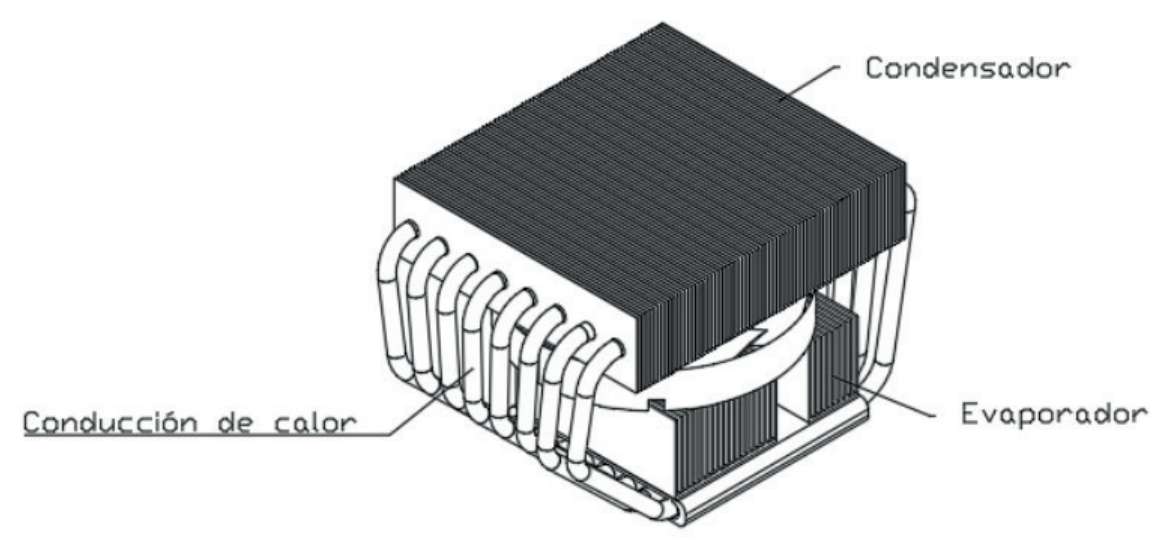

El dispositivo está formado por dos secciones, el evaporador (placa base de ancho 78.90 mm y largo $78.90 \mathrm{~mm}$ ) y el condensador (aletas de $0.38 \mathrm{~mm}$ de espesor) las cuales están dise- 
ñadas en aluminio con la finalidad de aumentar el área superficial para la transferencia de calor mediante tubos de calor de cobre, los cuales tienen una sección transversal de $6.61 \mathrm{~mm}$ de diámetro, como se aprecia en la Figura 2.

Figura 2. Dimensiones generales del disipador de calor
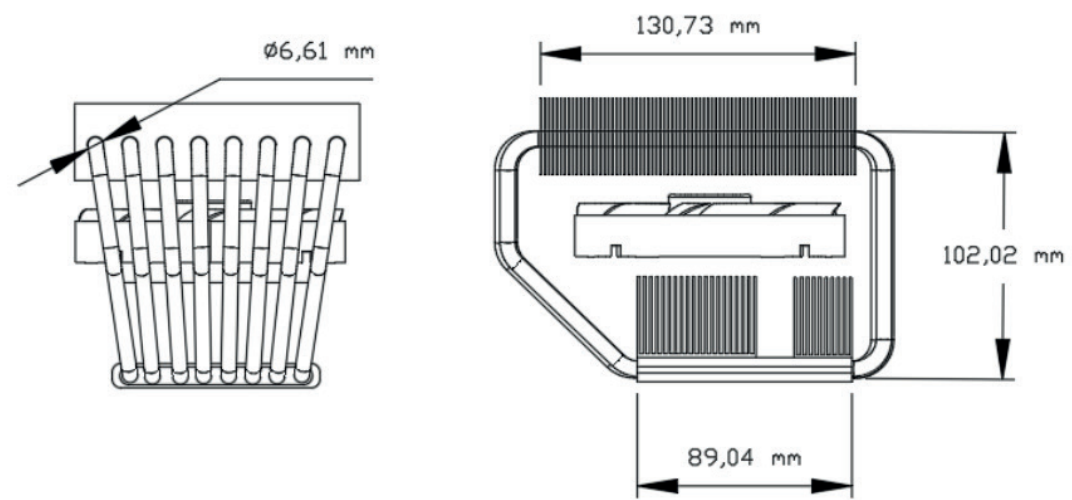

\subsection{Estudio térmico de disipación de calor}

El estudio de la conducción y convección de los componentes sólidos del disipador se realizó en ANSYS con Steady-state Thermal; en la Figura 3 se aprecia el mallado del dispositivo, se realiza una malla adaptiva con 186137 nodos y 29990 elementos. En el diseño de los elementos se utiliza aluminio 1050A para las aletas alrededor de los tubos que funcionan como condensador, así como para los radiadores que ayudan en el proceso de evaporación; los tubos de calor son diseñados de cobre. Las condiciones del ambiente se fijan a $25^{\circ} \mathrm{C}$. Además, se ha simulado una convección natural de los elementos hacia el ambiente compuesto de aire y convección forzada inducida por el ventilador en la zona de condensación. La convección forzada en las aletas es generada por un ventilador axial, en el cual el aire que fluye alcanza velocidades de $8 \mathrm{~m} / \mathrm{s}$ provocando una convección de $100 \mathrm{~W} / \mathrm{m}^{2}$.

Figura 3. Mallado del disipador de calor en el software ANSYS

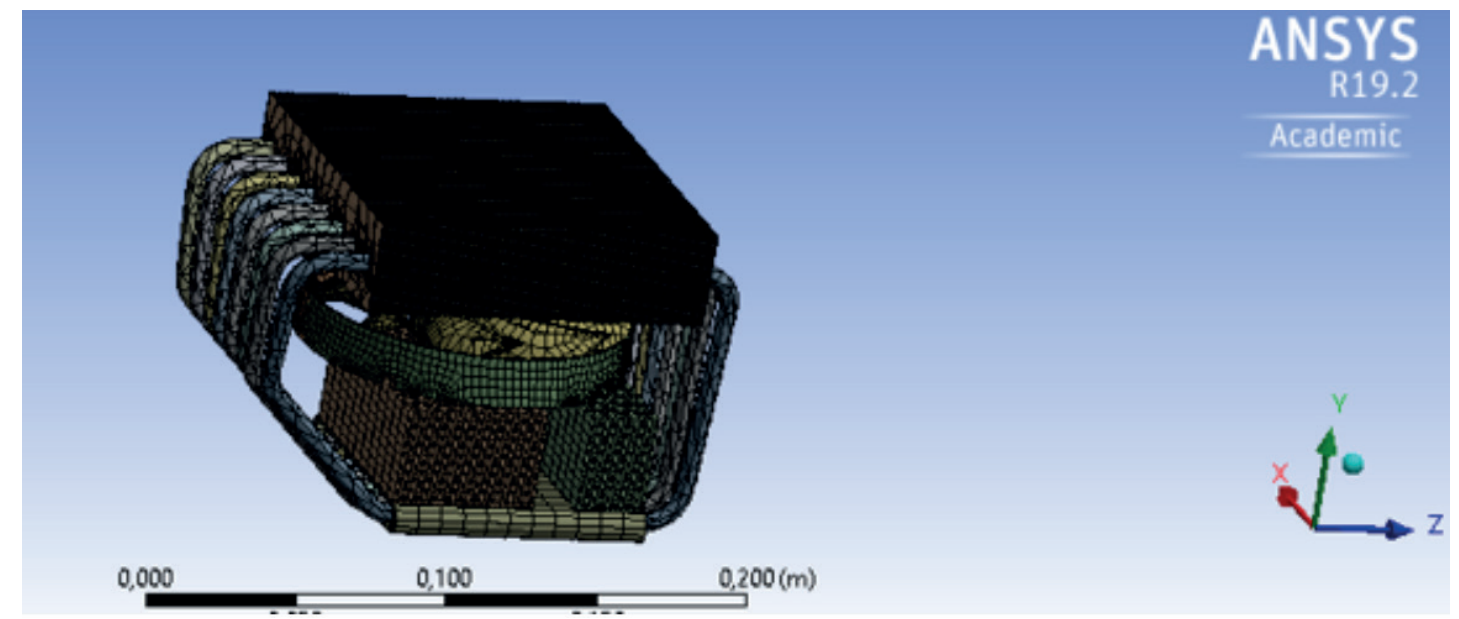


Las pruebas se realizaron a las temperaturas críticas con las que puede trabajar un procesador de alto desempeño (entre 80 y $90^{\circ} \mathrm{C}$ ), para analizar el diferencial de temperatura y el flujo de calor a través del disipador, reduciendo la temperatura del procesador al que está conectado.

Para el análisis de los tubos de calor que forman parte de la transferencia de calor del disipador, se evalúa con Fluent (CFD) de ANSYS 19.2, en 3D y con doble precisión, en este estudio se analizan: la transferencia de calor como el cambio de temperatura y la rapidez de evaporación y condensación del fluido a través del tiempo, para la simulación se utilizan los fluidos de trabajo anteriormente descritos, en la Tabla 1 se observan las presiones con las que se evalúan los fluidos de trabajo.

Tabla 1. Presión de trabajo de los fluidos

\begin{tabular}{|l|l|}
\hline \multicolumn{1}{|c|}{ Fluido de trabajo } & \multicolumn{1}{c|}{ Presión de trabajo (kPa) } \\
\hline R134a & 800 \\
\hline Agua & 70 \\
\hline Metanol & 59 \\
\hline
\end{tabular}

Para el análisis del tubo de calor se configuran los siguientes modelos: Multiphase Model con dos fases eulerianas para simular el cambio de fase de líquido a vapor, Viscous Model con un análisis K- épsilon estándar con tratamiento de pared, mejorado para simular los efectos térmicos y una corrección de curvatura, además un modelo de energía activo.

Las ecuaciones masa, conservación de momento y energía para el modelo multifásico euleriano se describen a continuación.

La ecuación de la continuidad para la fase q es:

$$
\frac{\partial}{\partial t}\left(\alpha_{q} \rho_{q}\right)+\nabla \cdot\left(\alpha_{q} \rho_{q} \vec{V}_{q}\right)=\sum_{p=1}^{n}\left(\dot{m}_{p q}-\dot{m}_{q p}\right)+S_{q}
$$

Donde, $\vec{V}_{q}$ es la velocidad de la fase q y $\dot{m}_{p q}$ caracteriza la transferencia de masa de la fase $\mathrm{p}$ a la fase $\mathrm{q}$.

El balance de momento para los campos de la fase q se determinan con la siguiente ecuación:

$$
\begin{aligned}
& \frac{\partial}{\partial t}\left(\alpha_{q} \rho_{q}\right)+\nabla \cdot\left(\alpha_{q} \rho_{q} \vec{V}_{q} \vec{V}_{q}\right)=-\alpha_{q} \nabla \mathrm{p}+\nabla \cdot \overline{\bar{\tau}}_{q}+\alpha_{q} \rho_{q} \vec{g}+\sum_{n=1}^{n}\left(\vec{R}_{p q}+\dot{m}_{p q} \vec{V}_{p q}-\dot{m}_{q p} \vec{V}_{q p}\right) \\
& +\left(\vec{F}_{q}+\vec{F}_{l i f t, q}+\vec{F}_{w l, q}+\vec{F}_{v m, q}+\vec{F}_{t d, q}\right)
\end{aligned}
$$

Donde, $\overline{\bar{\tau}}_{q}$ es el tensor de deformación para la fase q; $\vec{F}_{q}$ es una fuerza externa del cuerpo; $\vec{F}_{l i f t, q}$ es una fuerza de elevación; $\vec{F}_{w l, q}$ es la fuerza de lubricación de la pared; $\vec{F}_{v m, q}$ es una fuerza de masa virtual; $\vec{F}_{t d, q}$ es una fuerza de dispersión turbulenta, $\vec{R}_{p q}$ es la fuerza de interacción entre fases y $p$ es la presión compartida por todas las fases.

Para describir la conservación de energía en las aplicaciones multifásicas con el enfoque de Euler, se escribe la ecuación de entalpía para cada fase.

$$
\frac{\partial}{\partial t}\left(\alpha_{q} \rho_{q} h_{q}\right)+\nabla \cdot\left(\alpha_{q} \rho_{q} \vec{u}_{q} h_{q}\right)
$$




$$
=\alpha_{q} \frac{\partial p_{q}}{\partial t}+\overline{\bar{\tau}}_{q} \cdot \nabla \vec{u}_{q}-\nabla \vec{q}_{q}+S_{q}+\sum_{n}^{p=1}\left(Q_{p q}+\dot{m}_{p q} h_{p q}-\dot{m}_{q p} h_{q p}\right)-\nabla \cdot \sum_{j} h_{j, q} \vec{\jmath}_{j, q}
$$

Donde, $h_{q}$ es la entalpía específica de la fase; $\vec{q}_{q}$ es el flujo de calor; $S_{q}$ es un término que incluye fuentes de entalpía; $Q_{p q}$ es la intensidad del intercambio de calor entre las fases; $h_{p q}$ es la entalpía de la interfase; $h_{j, q}$ es la entalpía de las especies en la fase; $\vec{\jmath}_{j, q}$ flujo difusivo de las especies en fase.

Por otro lado, los modelos de turbulencia han sido útiles en cálculos prácticos, el modelo k-є estándar es un modelo semi-empírico basado en ecuaciones de transporte modelo para la energía cinética de turbulencia $(k)$ y su tasa de disipación $(\varepsilon)$. La ecuación de transporte modelo para $k$ se deriva de la ecuación exacta, mientras que la ecuación de transporte modelo para $\varepsilon$ se obtuvo utilizando el razonamiento físico. En la derivación del modelo k-e, se consideró que el flujo es completamente turbulento, y los efectos de la viscosidad molecular son insignificantes (Bardina, Huang, y Coakley, 1997). El modelo k - $\varepsilon$ estándar es por lo tanto válido solo para flujos turbulentos.

La energía cinética de la turbulencia $k$, y su velocidad de disipación $\boldsymbol{\varepsilon}$, se obtienen a partir de las siguientes ecuaciones de transporte:

$$
\begin{aligned}
& \frac{\partial}{\partial}(\rho k)+\frac{\partial}{\partial x_{i}}\left(\rho k u_{i}\right)=\frac{\partial}{\partial x_{j}}\left[\left(\mu+\frac{u_{t}}{\sigma_{k}}\right) \frac{\partial k}{\partial x_{j}}\right]+G_{k}+G_{b}-\rho \varepsilon-Y_{M}+S_{k} \\
& \frac{\partial}{\partial t}(\rho \varepsilon)+\frac{\partial}{\partial x_{i}}\left(\rho \varepsilon u_{i}\right)=\frac{\partial}{\partial x_{j}}\left[\left(\mu+\frac{u_{t}}{\sigma_{\varepsilon}}\right) \frac{\partial \varepsilon}{\partial x_{j}}\right]+C_{1 \varepsilon} \frac{\varepsilon}{k}\left(G_{k}+C_{3 \varepsilon} G_{b}\right)-\rho C_{2 \varepsilon} \frac{\varepsilon^{2}}{k}+S_{\varepsilon}
\end{aligned}
$$

Donde, $G_{k}$ representa la generación de energía cinética de turbulencia debido a los gradientes de velocidad promedio. $G_{\boldsymbol{b}}$ es la generación de energía cinética de turbulencia debido a la flotabilidad. La cantidad $Y_{M}$ simboliza la contribución de la dilatación de la fluctuación a la turbulencia incompresible a la tasa de disipación global. $C_{1 \varepsilon}, C_{2 \varepsilon}$ y $C_{3 \varepsilon}$ son constantes. $\sigma_{k}$ y $\sigma_{\varepsilon}$

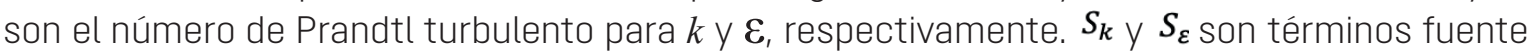
definidos por el usuario.

\section{Resultados y discusión}

En la simulación numérica, utilizando el software computacional, se determinan diversas variables del proceso de refrigeración utilizando tubos de calor, estas son: temperatura, fracción volumétrica y el coeficiente de transferencia de calor para los tres fluidos de análisis.

La temperatura representa la variable del proceso más importante en este estudio, debido a que se trata de obtener una temperatura de diseño del tubo de calor por donde circula el fluido que hará como agente refrigerante. El calor que se va a rechazar del equipo electrónico se toma a una temperatura de $90{ }^{\circ} \mathrm{C}$ en donde se encuentra el evaporador, y disipándola en la zona baja del condensador. La superficie exterior del tubo está expuesta a un ambiente cuya temperatura es de $25^{\circ} \mathrm{C}$.

En la Figura 4 se presentan resultados favorables en cuanto al diferencial de temperatura a lo largo del tubo disipador de calor al utilizar agua como fluido de trabajo, lo que se traduce en un rápido transporte de calor desde el evaporador al condensador. 
Figura 4. Temperatura del tubo de calor utilizando agua: a) 1 segundo, b) 5 segundos

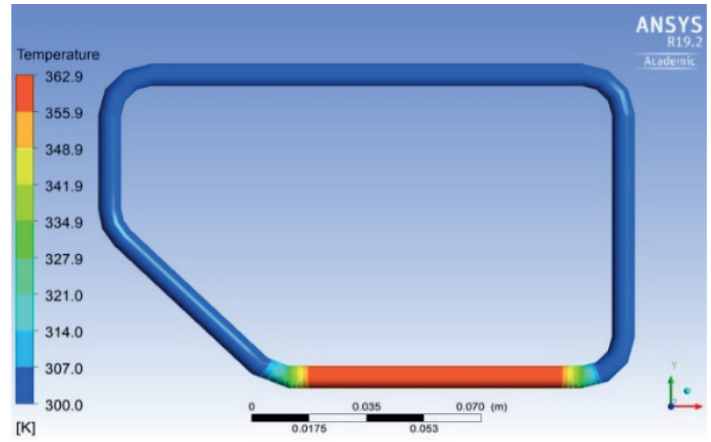

a)

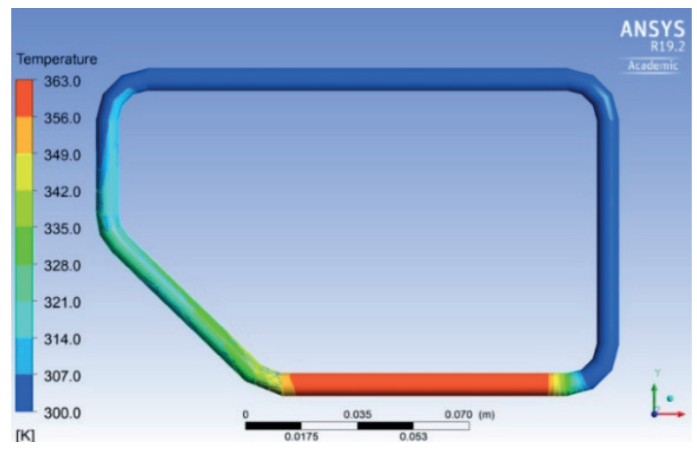

b)

Al utilizar otro fluido en el interior del tubo de calor como el metanol, se aprecia en la Figura 5 que, la temperatura no aumenta tanto como con el agua, sin embargo, existen cambios importantes en la conducción de calor.

Figura 5. Temperatura del tubo de calor utilizando metanol: a) 1 segundo, b) 5 segundos

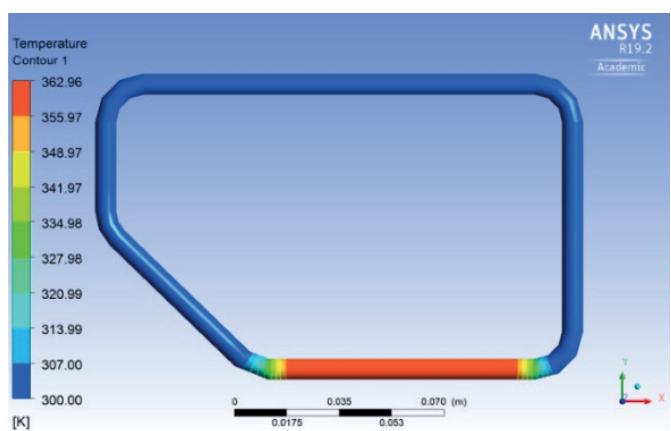

a)

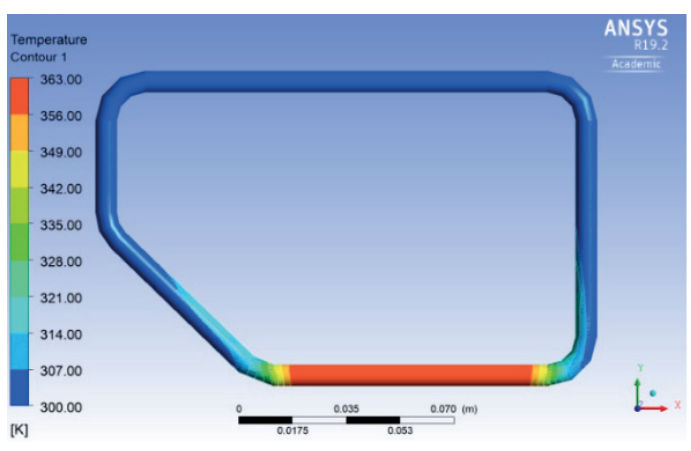

b)

El refrigerante 134a muestra una distribución uniforme de temperatura en la zona inclinada del tubo, lo cual se esperaba para esta estructura de diseño de tubo de calor, esto se aprecia en la Figura 6.

Figura 6. Temperatura del tubo de calor utilizando R134a: a) 1 segundo, b) 5 segundos

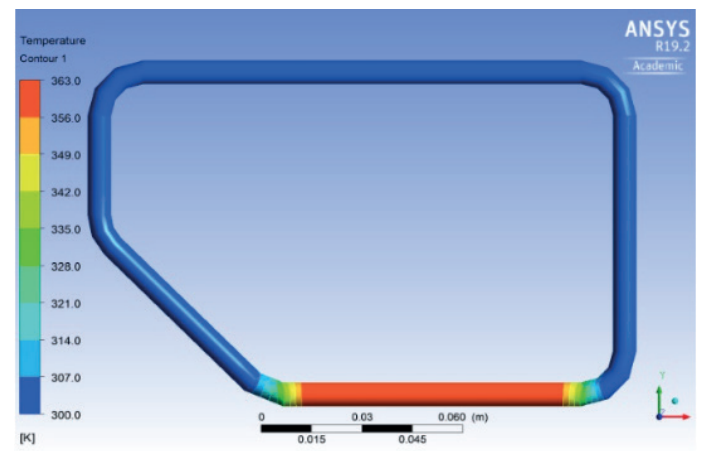

a)

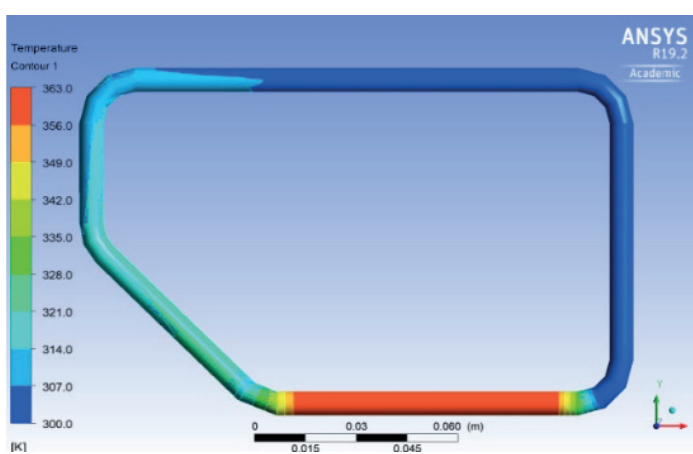

b) 
En una comparación entre los tres fluidos (Figura 7), se observa el diferente rendimiento a los 5 s de comenzada la disipación, en cuanto al transporte del calor a través del tubo de calor por parte de cada fluido estudiado.

Figura 7. Cambios de temperatura en las zonas del tubo de calor debido al transporte de calor

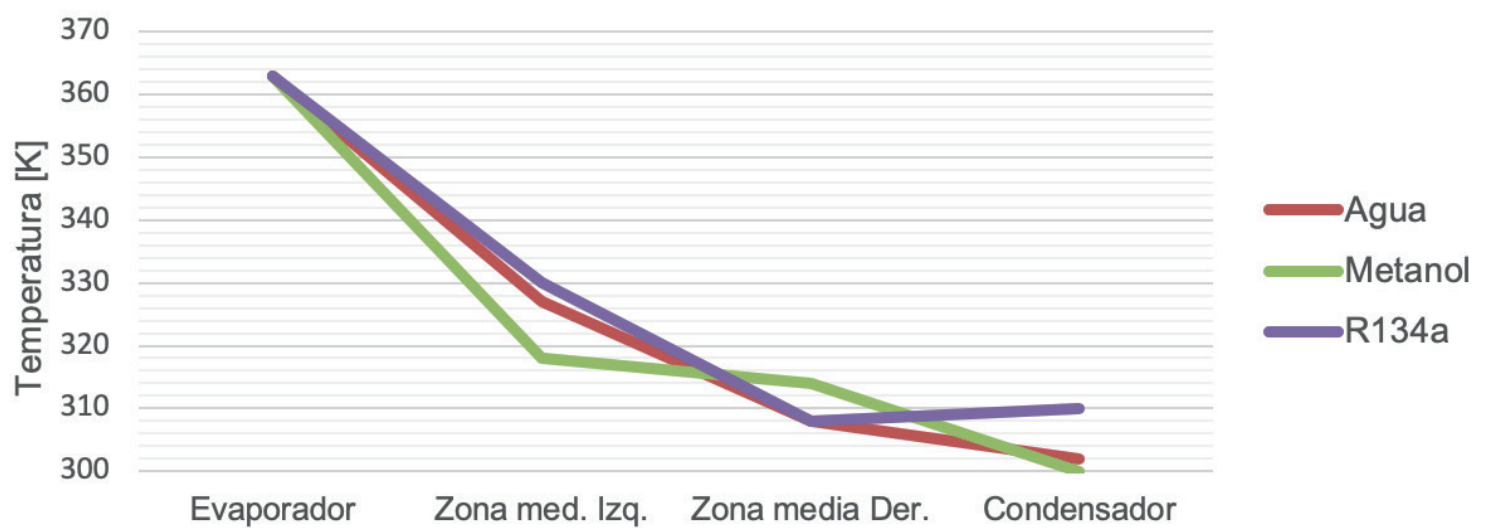

La otra variable importante en esta investigación es el coeficiente de transferencia de calor que el fluido tiene sobre el tubo de calor y este a su vez en los equipos electrónicos que poseen alta temperatura.

En la Figura 8, se aprecia el coeficiente de transferencia de calor para el fluido agua, este va aumentando a medida que gana calor desde las superficies calientes y se lo relaciona en función del tiempo.

Figura 8. Coeficiente de transferencia de calor del agua: a) 1 segundo, b) 5 segundos

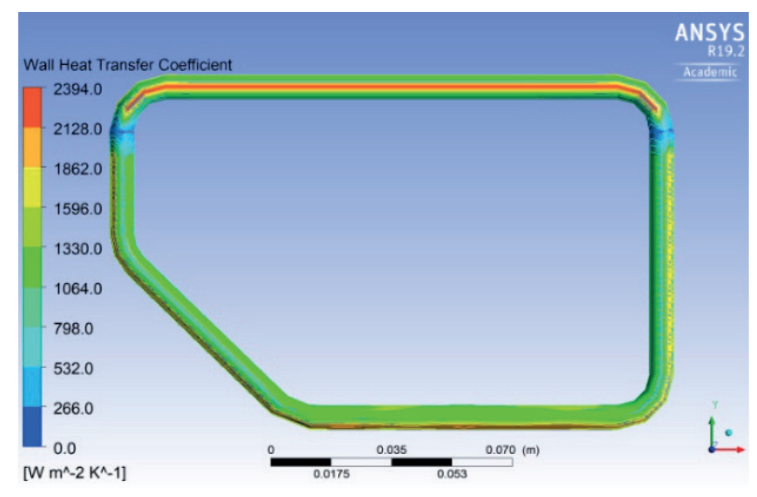

a)

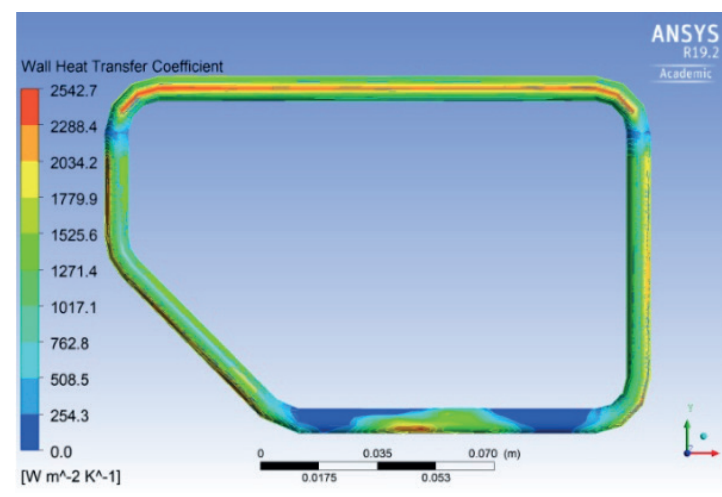

b)

El coeficiente de transferencia de calor del metanol se visualiza en la Figura 9, por los procesos de evaporación y condensación que sufre el fluido, el comportamiento del coeficiente de transferencia es parecido al del agua, sin embargo, al pasar el tiempo los valores disminuyen notablemente. 
Figura 9. Coeficiente de transferencia de calor del metanol: a) 1 segundo, b) 5 segundos

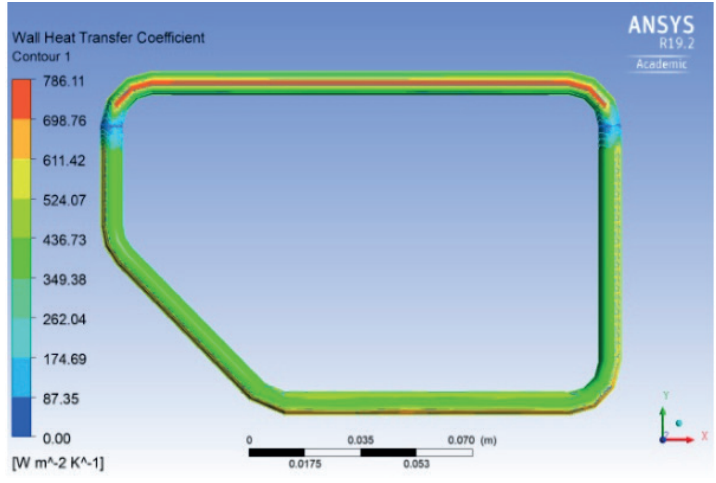

a)

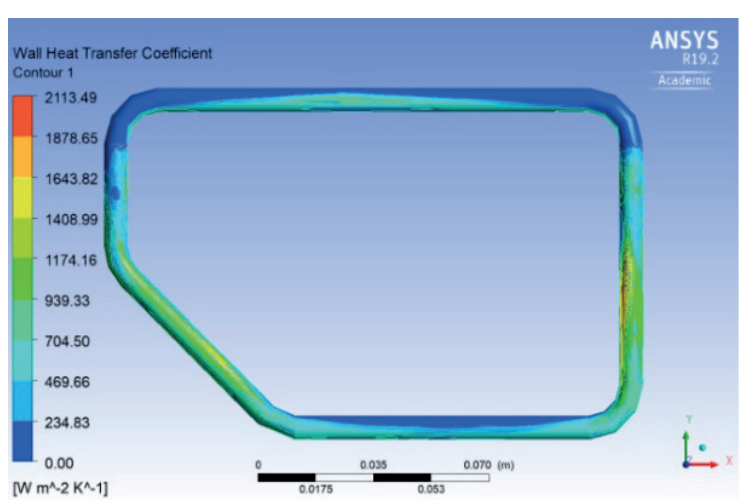

b)

El coeficiente de transferencia de calor del refrigerante 134a varía con el tiempo y el gradiente de los coeficientes no es tan grande en comparación con los mostrados para el agua y metanol, esto se observa en la Figura 10.

Figura 10. Coeficiente de transferencia de calor del R134a: a) 1 segundo, b) 5 segundos

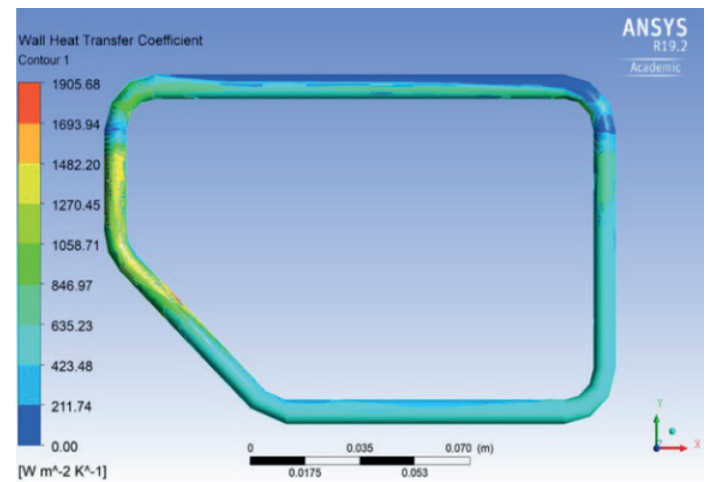

a)

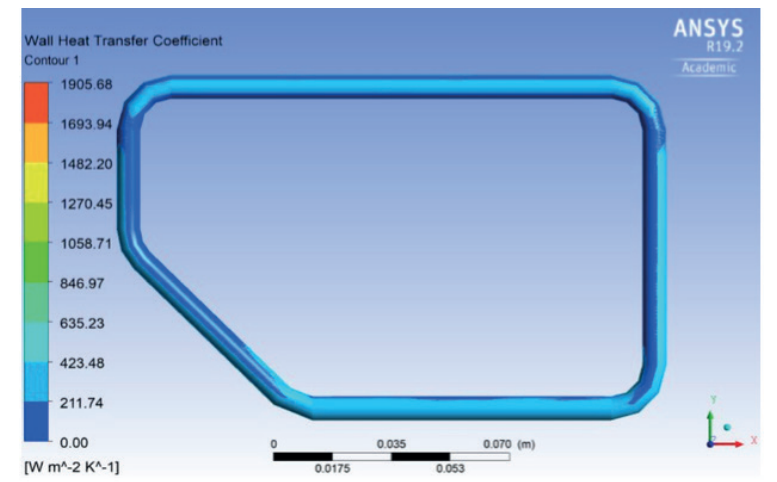

b)

En la Figura 11 se cuantifica los máximos valores de transferencia de calor alcanzados por cada fluido durante el tiempo que se realizó la prueba, en donde se puede apreciar que el agua genera un mejor desempeño en la transferencia de calor.

Figura 11. Gráfica comparativa del coeficiente de transferencia de calor $\left(\mathrm{W} / \mathrm{m}^{2} \mathrm{~K}\right)$

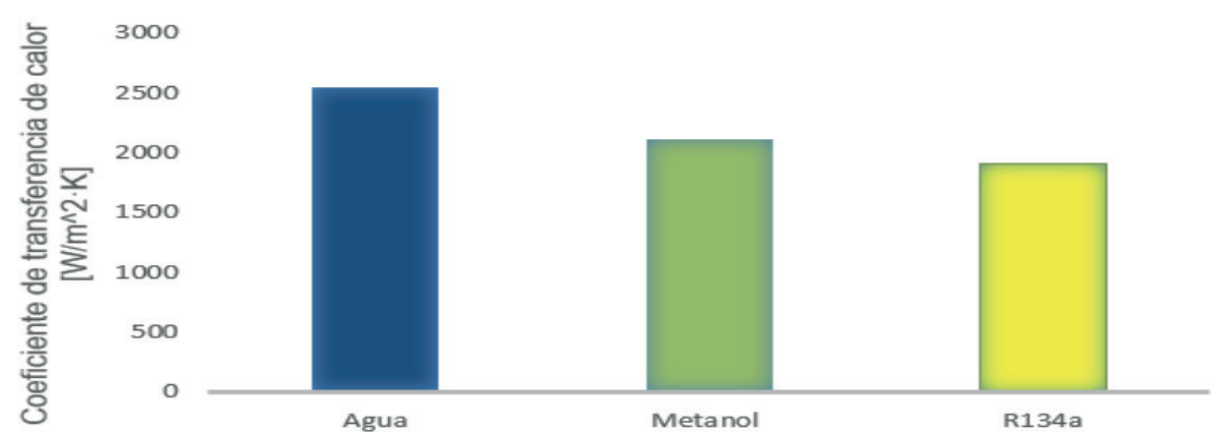


Por último, el determinar el cambio de fase que ocurre en el disipador de calor es de vital importancia en esta investigación, debido a que, con esta variable se observa cómo se comportan los fluidos en el proceso de rechazo de calor.

En la Figura 12, se observa fácilmente el cambio de fase del agua al estar en contacto con la fuente de calor, al inicio en un tiempo determinado de 1 segundo, así como también a los cinco segundos, teniendo en cuenta que en la parte inferior se encuentra el evaporador, el cambio de fase se produce de forma total en ese espacio.

Figura 12. Cambio de fase del agua: a) 1 segundo, b) 5 segundos

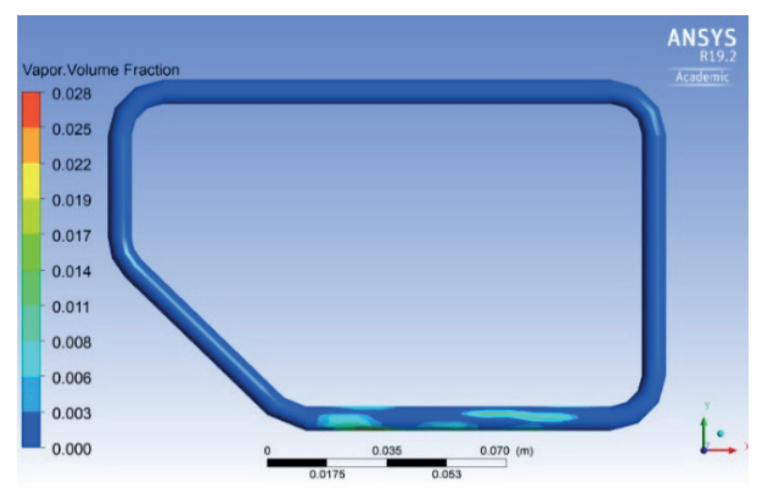

a)

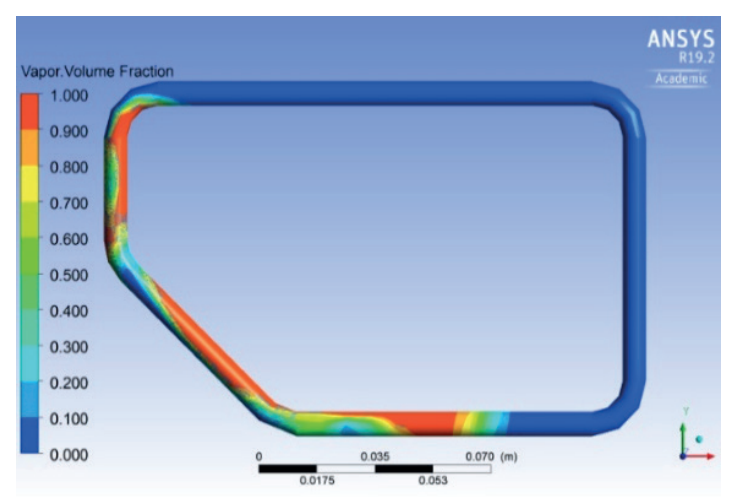

b)

El metanol presenta una evaporación rápida, el vapor asciende a la zona deseada correctamente por las diferencias de densidades entre fases, pero el vapor no fluye de manera constante hacia el condensador lo que podría demorar la condensación y retrasar el ciclo continuo de cambio de fase, esto es visible en la Figura 13

Figura 13. Cambio de fase del metanol: a) 1 segundo, b) 5 segundos

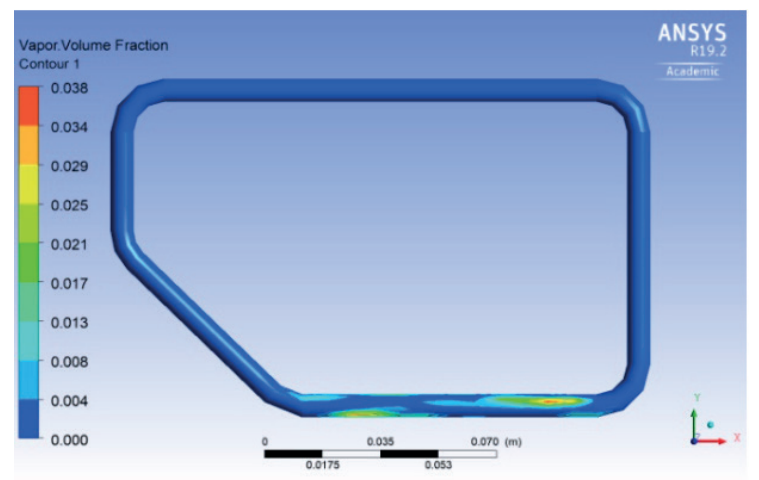

a)

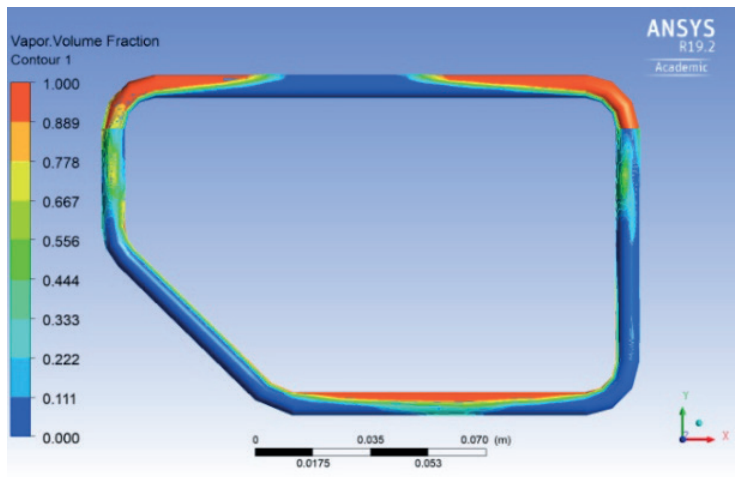

b)

Finalmente, al utilizar R134a como fluido de trabajo se visualiza (Figura 14), que no existe un cambio de fase importante dentro del tubo de calor, por lo que este fluido no sería el adecuado de usar en el interior del tubo. 
Figura 14. Cambio de fase del R134a: a) 1 segundo, b) 5 segundos

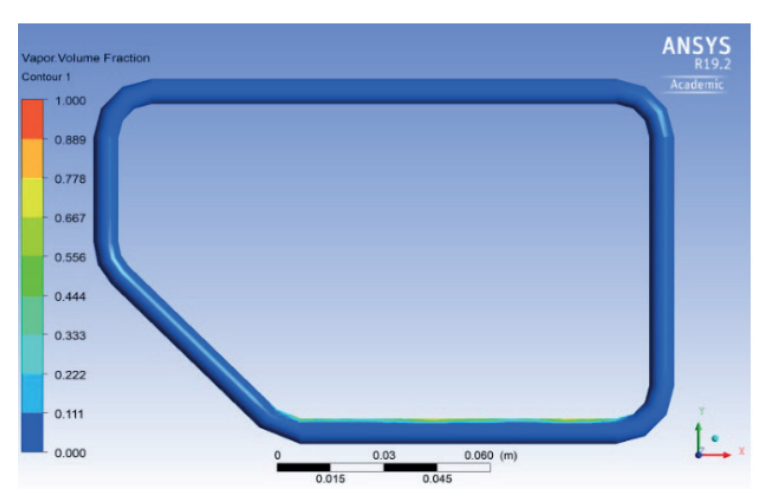

a)

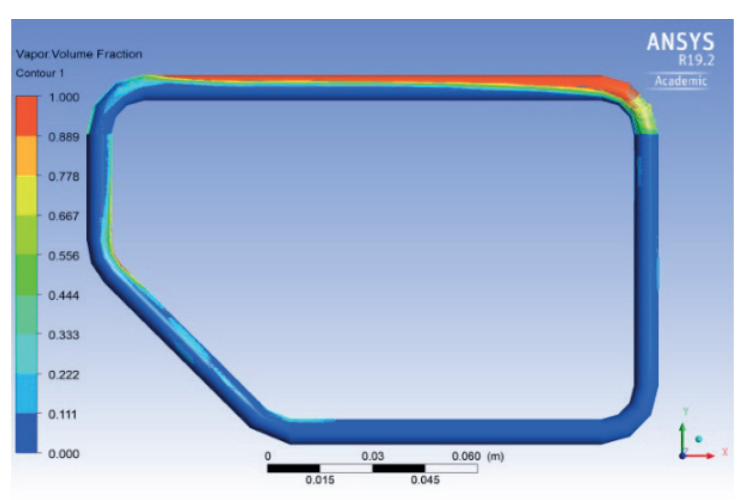

b)

\section{Conclusiones y recomendaciones}

De esta investigación se desprenden varias conclusiones las mismas que se detallan a continuación:

La utilización de tubos de calor en un disipador aumenta considerablemente la transferencia de calor y la velocidad de disipación, permitiendo enfriar de manera adecuada procesadores de alto desempeño, en las pruebas realizadas se obtiene un aumento considerable en la transferencia de calor hacia el ambiente de parte de cada tubo de calor, la conducción de calor muestra una mejora de hasta un $3.3 \%$ y ya que cumple un ciclo continuo de evaporación-condensación, la disipación de calor es más rápida hasta un 8 \% ya que, se aprovecha la absorción del calor latente necesario para el cambio de fase del fluido.

El agua presenta los mejores resultados para las pruebas térmicas realizadas en cuanto a conducción y coeficiente de transferencia de calor, seguido por el metanol el cual podría ser un sustituto muy favorable, presentando apenas un $2 \%$ menor en efectividad de transferencia de calor, además este presenta los mejores resultados en cuanto a rapidez de evaporación, en las pruebas muestra el mayor porcentaje de fluido evaporado con un $26 \%$. El refrigerante R134a, a pesar de mostrar los resultados menos favorables debido a las reducidas dimensiones del tubo, es otro candidato importante para el diseño de disipadores con tubos de calor, ya que se encontró un aumento en la conducción del calor favorable.

En el caso de la implementación del disipador en procesadores de bajo rendimiento, se recomienda utilizar aire dentro de los tubos de calor como fluido de transferencia, debido a que no se generan elevadas temperaturas, por lo cual este fluido es recomendable por que la conducción de calor es lenta.

\section{Agradecimientos}

Los autores agradecen de forma cordial al grupo de investigación en Energías Renovables e Implementación Mecánica de pymes de la Universidad Politécnica Salesiana, Quito-Ecuador, por sus grandes aportes a esta investigación. 


\section{Bibliografía}

Bardina, J., Huang, P., y Coakley, T. (1997). Turbulence modeling validation, testing, and development. NASA Technical Memorandum (110446), 1-88.

Blet, N., Lips, S., y Sartre, V. (2017). Heats pipes for temperature homogenization: A literature review. Applied Thermal Engineering, 118, 490-509. https://doi.org/10.1016/j.applthermaleng.2017.03.009

Chan, C. W., Siqueiros, E., Ling-Chin, J., Royapoor, M., y Roskilly, A. P. (2015). Heat utilisation technologies: A critical review of heat pipes. Renewable and Sustainable Energy Reviews, 50, 615-627. https:// doi.org/10.1016/j.rser.2015.05.028

Chen, X., Ye, H., Fan, X., Ren, T., y Zhang, G. (2016). A review of small heat pipes for electronics. Applied Thermal Engineering, 96, 1-17. https://doi.org/10.1016/j.applthermaleng.2015.11.048

Dillig, M., Leimert, J., y Karl, J. (2014). Planar high temperature heat pipes for SOFC/SOEC stack applications. Fuel Cells, 14(3), 479-488. https://doi.org/10.1002/fuce.201300224

Dinker, A., Agarwal, M., y Agarwal, G. D. (2017). Heat storage materials, geometry and applications: A review. Journal of the Energy Institute, 90(1), 1-11. https://doi.org/10.1016/j.joei.2015.10.002

Faghri, A. (2014). Heat Pipes: Review, Opportunities and Challenges. Frontiers in Heat Pipes, 5(1). https:// doi.org/10.5098/fhp.5.1

Garc, P. (2018). Control por modos deslizantes de un sistema de intercambio de calor : validación experimental (Sliding modes control for a heat Exchange system : experimental validation). Enfoque UTE, 110-119.

Garro, S., Díaz, L. A., Liang, J., Martínez, F., Meneses, W., Ortega, H., ... Stradi, B. (2012). Modelación y simulación de disipadores de calor para procesadores de computadora en COMSOL Multiphysics. Tecnología En Marcha, 25(3), 70-80.

Hung, Y. M., y Seng, Q. (2011). Effects of geometric design on thermal performance of star-groove microheat pipes. International Journal of Heat and Mass Transfer, 54(5-6), 1198-1209. https://doi. org/10.1016/j.ijheatmasstransfer.2010.09.070

Jafari, D., Franco, A., Filippeschi, S., y Di Marco, P. (2016). Two-phase closed thermosyphons: A review of studies and solar applications. Renewable and Sustainable Energy Reviews, 53, 575-593. https://doi.org/10.1016/j.rser.2015.09.002

Jafari, D., Shamsi, H., Filippeschi, S., Di Marco, P., y Franco, A. (2017). An experimental investigation and optimization of screen mesh heat pipes for low-mid temperature applications. Experimental Thermal and Fluid Science, 84, 120-133. https://doi.org/10.1016/j.expthermflusci.2017.02.005

Khalifa, A., Tan, L., Date, A., y Akbarzadeh, A. (2015). Performance of suspended finned heat pipes in hightemperature latent heat thermal energy storage. Applied Thermal Engineering, 81, 242-252. https://doi.org/10.1016/j.applthermaleng.2015.02.030

Lee, J., y Kim, S. J. (2017). Effect of channel geometry on the operating limit of micro pulsating heat pipes. International Journal of Heat and Mass Transfer, 107, 204-212. https://doi.org/10.1016/j.ijheatmasstransfer.2016.11.036

Lee, S., Pandiyan, D., Seo, J. S., Phelan, P. E., y Wu, C. J. (2016). Thermoelectric-based sustainable self-cooling for fine-grained processor hot spots. Proceedings of the 15th InterSociety Conference on Thermal and Thermomechanical Phenomena in Electronic Systems, ITherm 2016, 847-856. https://doi.org/10.1109/ITHERM.2016.7517635

Orr, B., Akbarzadeh, A., Mochizuki, M., y Singh, R. (2016). A review of car waste heat recovery systems utilising thermoelectric generators and heat pipes. Applied Thermal Engineering, 101, 490-495. https://doi.org/10.1016/j.applthermaleng.2015.10.081

Reay, D., y Harvey, A. (2013). The role of heat pipes in intensified unit operations. Applied Thermal Engineering, 57(1-2), 147.153. https://doi.org/10.1016/j.applthermaleng.2012.04.002

Shabgard, H., Allen, M. J., Sharifi, N., Benn, S. P., Faghri, A., y Bergman, T. L. (2015). Heat pipe heat exchangers and heat sinks: Opportunities, challenges, applications, analysis, and state of the art. 
International Journal of Heat and Mass Transfer, 89, 138-158. https://doi.org/10.1016/j.ijheatmasstransfer.2015.05.020

Tang, H., Tang, Y., Wan, Z., Li, J., Yuan, W., Lu, L., ... Tang, K. (2018). Review of applications and developments of ultra-thin micro heat pipes for electronic cooling. Applied Energy, 223(mayo), 383-400. https://doi.org/10.1016/j.apenergy.2018.04.072

Velasco Roldán, L., Goyos Pérez, L., Delgado García, R., y Freire Amores, L. (2017). Instalación para medición de conductividad térmica en composites basados en residuos de biomasa. Enfoque UTE, 7(2), 69. https://doi.org/10.29019/enfoqueute.v7n2.96 